\title{
A NOTE ON LIE ALGEBRA COHOMOLOGY
}

\author{
MICHAEL J. LARSEN AND VALERY A. LUNTS
}

\begin{abstract}
Given a finite dimensional Lie algebra $L$ let $I$ be the augmentation ideal in the universal enveloping algebra $U(L)$. We study the conditions on $L$ under which the Ext-groups $\operatorname{Ext}(k, k)$ for the trivial $L$-module $k$ are the same when computed in the category of all $U(L)$ modules or in the category of $I$-torsion $U(L)$-modules. We also prove that the Rees algebra $\oplus_{n \geq 0} I^{n}$ is Noetherian if and only if $L$ is nilpotent. An application to cohomology of equivariant sheaves is given.
\end{abstract}

\section{INTRODUCTION}

Let $L$ be a finite dimensional Lie algebra over a field $k$. Consider the universal enveloping algebra $U(L)$ with the augmentation ideal $I \subset U(L)$. Denote by $U(L)$-mod the category of finitely generated left $U(L)$-modules and by $(U(L) \text {-mod })_{I} \subset U(L)$-mod the Serre subcategory of $I$-torsion modules. We have the obvious functor

$$
\Phi_{L}: D^{b}\left((U(L)-\bmod )_{I}\right) \rightarrow D_{I}^{b}(U(L) \text {-mod })
$$

where $D_{I}^{b}(U(L)$-mod $) \subset D^{b}(U(L)$-mod $)$ is the full triangulated subcategory consisting of complexes with $I$-torsion cohomology. In this paper we study the question:

Question. When is $\Phi_{L}$ an equivalence?

The functor $\Phi_{L}$ being an equivalence means that the Ext-groups $\operatorname{Ext}^{i}(k, k)$ for the trivial $L$-module $k$ are the same in the categories $U(L)$-mod and $(U(L)-\bmod )_{I}$.

We answer this question in Theorem 1.1 below.

Define inductively the decreasing sequence of ideals in $L$ :

$$
L_{1}=L, \quad L_{n}=\left[L, L_{n-1}\right]
$$

and put $L_{\infty}=\bigcap_{n} L_{n}$. This is an ideal in $L$ such that the quotient Lie algebra $L_{\text {nil }}:=L / L_{\infty}$ is nilpotent. We have $L_{\infty}=0$ if and only if $L$ is nilpotent.

For each $i$ the cohomology $H^{i}\left(L_{\infty}, k\right)$ is naturally an $L_{\text {nil-module. Denote }}$ by $H^{>0}\left(L_{\infty}, k\right)$ the positive degree cohomology.

ML was partially supported by NSF grant DMS-1702152. VL was partially supported by Laboratory of Mirror Symmetry NRU HSE, RF Government grant, ag. No. 14.641.31.0001. 
Theorem 1.1. The functor $\Phi_{L}$ is an equivalence if and only if the $L_{\text {nil- }}$ module $H^{>0}\left(L_{\infty}, k\right)$ has no subquotients isomorphic to the trivial module $k$. For example $\Phi_{L}$ is an equivalence if $L$ is nilpotent.

We find it natural to approach Theorem 1.1 by studying the graded Rees algebra

$$
U(L)^{*}:=\bigoplus_{n \geq 0} I^{n}=U(L) \oplus I \oplus I^{2} \oplus \cdots
$$

It is easy to prove the following result.

Proposition 1.2. If the algebra $U(L)^{*}$ is graded left Noetherian, then the functor $\Phi_{L}$ is an equivalence.

It is, however, not necessary for $U(L)^{*}$ to be graded left Noetherian in order for $\Phi_{L}$ to be an equivalence.

The next theorem may be of independent interest.

Theorem 1.3. The algebra $U(L)^{*}$ is graded left Noetherian if and only if $L$ is nilpotent.

In the last section of the paper we mention an application of Theorem 1.1 to the cohomology of quasi-coherent sheaves which are equivariant with respect to a unipotent group.

In this paper we consider only left modules, but all the results are also valid (with the same proofs) for right modules.

We fix a field $k$. All Lie algebras are finite dimensional over $k$. All associative rings are unital.

Acknowledgements. We thank Grigory Papayanov for a useful discussion.

\section{A CRITERION FOR EQUIVALENCE OF CATEGORIES}

Let $R$ be an associative left Noetherian ring with a 2 -sided ideal $I \subset R$. Let $M$ be a left $R$-module. An element $m \in M$ is called $I$-torsion, if $I^{n} m=0$ for some $n>0$. The collection of $I$-torsion elements in $M$ is an $R$-submodule, which we denote by $M_{I}$. We say that $M$ is torsion if $M_{I}=M$.

Let $R$-mod denote the abelian category of finitely generated left $R$-modules and let $(R \text {-mod })_{I} \subset R$-mod be its full Serre subcategory of $I$-torsion modules. Let $C^{b}\left(R\right.$-mod) (resp. $\left.C^{b}\left((R \text {-mod })_{I}\right)\right)$ be the category of bounded complexes over $R$-mod (resp. over $\left.(R \text {-mod })_{I}\right)$ and let $C_{I}^{b}(R$-mod $) \subset C^{b}(R$-mod $)$ be the full subcategory of complexes whose cohomology groups are torsion.

In the bounded derived category $D^{b}(R$-mod) consider the full subcategory $D_{I}^{b}(R$-mod $)$ of complexes with torsion cohomology groups. We have the obvious functor

$$
\Phi=\Phi_{R}: D^{b}\left((R-\bmod )_{I}\right) \rightarrow D_{I}^{b}(R \text {-mod })
$$

Proposition 2.1. Assume that for every finitely generated left $R$-module $M$ there exists a submodule $N \subset M$ such that $N_{I}=0$ and $M / N$ is I-torsion. Then the functor $\Phi$ is an equivalence. 
Proof. Let $A^{\bullet}$ be an object of $C_{I}^{b}(R$-mod). We claim there exists an object $B^{\bullet}$ of $C^{b}\left((R \text {-mod })_{I}\right)$ and a morphism of complexes $f: A^{\bullet} \rightarrow B^{\bullet}$ which is a quasi-isomorphism. Indeed, let

$$
A^{\bullet}=0 \rightarrow A^{i} \stackrel{d^{i}}{\longrightarrow} A^{i+1} \stackrel{d^{i+1}}{\longrightarrow} \cdots \stackrel{d^{n-1}}{\longrightarrow} A^{n} \rightarrow 0
$$

and let $t$ be the lowest index such that $A_{I}^{t} \neq A^{t}$. By assumption there exists a submodule $P \subset A^{t}$ such that $P_{I}=0$ and $\left(A^{t} / P\right)_{I}=A^{t} / P$. We claim that $P \cap$ ker $d^{t}=0$. Indeed, since $H^{t}\left(A^{\bullet}\right)$ and $A^{t-1}$ are torsion, it follows that ker $d^{t}$ is torsion, so $P \cap \operatorname{ker} d^{t}=0$. Therefore, the complex $A^{\bullet}$ contains an acyclic subcomplex $\tilde{P}:=P \stackrel{\sim}{\rightarrow} d^{t}(P)$ and the components with index $\leq t$ of the quotient complex $A^{\bullet} / \tilde{P}$ are torsion. Iterating this process we find the required quasi-isomorphism $f: A^{\bullet} \rightarrow B^{\bullet}$. This shows that the functor $\Phi$ is essentially surjective.

For complexes $C^{\bullet}, D^{\bullet}$ representing objects in $D^{b}\left((R \text {-mod })_{I}\right)$, a morphism $\Phi\left(D^{\bullet}\right) \rightarrow \Phi\left(C^{\bullet}\right)$ is represented by a diagram of complexes $D^{\bullet} \rightarrow A^{\bullet} \stackrel{s}{\leftarrow} C^{\bullet}$, where $A^{\bullet} \in C_{I}^{b}(R$-mod) and $s$ is a quasi-isomorphism. The fact that the functor $\Phi$ is full and faithful now follows, since (as shown above) there exists a complex $B^{\bullet} \in C^{b}\left((R \text {-mod })_{I}\right)$ and a morphism $f: A^{\bullet} \rightarrow B^{\bullet}$ of complexes that is a quasi-isomorphism.

Consider now the graded Rees algebra

$$
R^{*}:=\bigoplus_{n \geq 0} I^{n}=R \oplus I \oplus I^{2} \oplus \cdots
$$

Lemma 2.2. Assume that the algebra $R^{*}$ is graded left Noetherian (i.e. every graded left ideal is finitely generated). Then the assumption of Proposition 2.1] holds: for any finitely generated left $R$-module $M$ there exists a submodule $N \subset M$ such that $N_{I}=0$ and $M / N=(M / N)_{I}$

Proof. Let $M$ be a finitely generated $R$-module. If $M=M_{I}$, then we can take $N=0$. So assume that $M \neq M_{I}$, i.e. $I^{s} M \neq 0$ for all $s>0$. Consider the graded finitely generated $R^{*}$-module

$$
\tilde{M}=M \oplus I M \oplus I^{2} M \oplus \cdots
$$

and its graded submodule

$$
P=M_{I} \oplus\left(I M \cap M_{I}\right) \oplus\left(I^{2} M \cap M_{I}\right) \oplus \cdots
$$

By our assumption, $P$ is finitely generated; hence, there exists $n>0$ such that for all $m>0$

$$
I^{m}\left(I^{n} M \cap M_{I}\right)=I^{n+m} M \cap M_{I}
$$

As $I^{n} M \cap M_{I}$ is finitely generated and $I$-torsion, it is annihilated by $I^{m}$ for some $m$. Thus,

$$
\left(I^{m+n} M\right)_{I}=I^{m+n} M \cap M_{I}=I^{m}\left(I^{n} M \cap M_{I}\right)=0 .
$$

Putting $N=I^{m+n} M$, we have $N_{I}=0$ and $(M / N)_{I}=M / N$. 
Corollary 2.3. Assume that the algebra $R^{*}$ is graded left Noetherian. Then the functor $\Phi$ is an equivalence.

\section{When is the Rees algebra of a universal EnVEloping Algebra NOETHERIAN}

Let $L$ be a finite dimensional Lie algebra, $U(L)$ its universal enveloping algebra and $I \subset U(L)$ the augmentation ideal. As above, we consider the graded Rees algebra

$$
U(L)^{*}=\bigoplus_{n \geq 0} I^{n}=U(L) \oplus I \oplus I^{2} \oplus \cdots
$$

The main result of this section is the following theorem.

Theorem 3.1. The algebra $U(L)^{*}$ is graded left Noetherian if and only if the Lie algebra $L$ is nilpotent.

Before proving the theorem we formulate a useful corollary.

Corollary 3.2. Let $L$ be a nilpotent Lie algebra. Then the functor

$$
\Phi_{U(L)}: D^{b}\left((U(L)-m o d)_{I}\right) \rightarrow D_{I}^{b}(U(L) \text {-mod })
$$

is an equivalence.

Proof. This follows from Theorem 3.1 and Corollary 2.3.

Proof. The proof of the theorem will take several steps and will occupy the rest of the section.

3.1. Proof of the "if" direction. The universal enveloping algebra $U(L)$ has a standard increasing filtration which induces a similar filtration in the Rees algebra $U(L)^{*}$. We will prove that if $L$ is nilpotent, then the associated (double) graded algebra gr $U(L)^{*}$ is commutative finitely generated, hence Noetherian.

So assume for now that the Lie algebra $L$ is nilpotent.

We define $L^{m}$ for positive integers $m$ recursively; $L^{1}:=L$, and

$$
L^{d}:=\sum_{j=1}^{d-1}\left[L^{j}, L^{d-j}\right]
$$

for $m \geq 2$. As $L$ is nilpotent, $L^{d}=\{0\}$ for all $d$ sufficiently large. We choose an ordered basis $\left\{e_{1}, \ldots, e_{n}\right\}$ of $L$ adapted to the decreasing filtration $L^{\bullet}$ in the sense that there exists a non-decreasing sequence $\nu_{1} \leq \ldots \leq \nu_{n}$ such that $\left\{e_{i} \mid \nu_{i} \geq d\right\}$ spans $L^{d}$ for all $d$. Thus, we can write

$$
\left[e_{i}, e_{j}\right]=\sum_{\left\{k \mid \nu_{k} \geq \nu_{i}+\nu_{j}\right\}} c_{k} e_{k} .
$$

Write for short $U:=U(L)$. We recall the standard increasing filtration $\left\{U_{r}\right\}_{r \geq 0}$ of $U$ for which $U_{r}$ is the span of all products $x_{1} x_{2} \cdots x_{r}$, where $x_{i} \in L \subset U$. For every multi-index $a=\left(a_{1}, \ldots, a_{n}\right)$ of non-negative integers, 
we denote by $e^{a}$ the monomial $e_{1}^{a_{1}} \cdots e_{n}^{a_{n}}$. Thus, $e^{a} \in U_{|a|}$, where $|a|:=$ $a_{1}+\cdots+a_{n}$. For every $x \in U$, we write $\operatorname{deg} x=0$ if $x \in U_{0}$ and $\operatorname{deg} x=r$ if $x \in U_{r} \backslash U_{r-1}$ for $r \geq 1$. We call this the degree of $x$.

The PBW theorem implies that for all $r \in \mathbb{N}$ the image of the set

$$
\left\{e^{a}:|a|=r\right\} \subset U_{r}
$$

in $U_{r} / U_{r-1}$ is a basis. Thus, the set of monomials $e^{a}$ as $a$ ranges over $\mathbb{N}^{n}$ is a basis of $U$, which we call the standard basis, and $\operatorname{deg} \sum_{a} c_{a} e^{a}$ is the largest value of $|a|$ for which $c_{a} \neq 0$. For each $r, U_{r}$ is spanned by $\left\{e^{a}:|a| \leq r\right\}$. Moreover, for all $x, y \in U$,

$$
\operatorname{deg}(x+y) \leq \max (\operatorname{deg} x, \operatorname{deg} y) .
$$

By the PBW theorem, if $x$ and $y$ are non-zero elements of $U$, then

$$
\operatorname{deg} x y=\operatorname{deg} x+\operatorname{deg} y .
$$

Let $I$ denote the augmentation ideal of $U$, or equivalently, the span of $e^{a}$ for all $a$ with $|a| \geq 1$.

Lemma 3.3. For all positive integers $m, I^{m}$ has basis

$$
\left\{e^{a} \mid \sum_{i} a_{i} \nu_{i} \geq m\right\}
$$

Proof. This set is a subset of the standard basis, so it is linearly independent. For $0<k<d,\left[L^{d-k}, L^{k}\right] \subset L^{d}$, so by induction on $d$, every $e_{i} \in L^{d}$ lies in $I^{d}$. Thus, the set (3.4) is contained in $I^{m}$.

We prove by induction that every element of $I^{m}$ is a linear combination of elements of (3.4), the case $m=1$ being trivial. It suffices to prove that if $i_{0}, \ldots, i_{k} \in\{1,2, \ldots, n\}, i_{1} \leq i_{2} \leq \cdots \leq i_{k}$, and

$$
\nu_{i_{1}}+\cdots+\nu_{i_{k}} \geq m-1,
$$

then $e_{i_{0}} e_{i_{1}} \cdots e_{i_{k}}$ lies in the span of (3.4) for $m$. We prove more precisely that for any sequence $i_{0}, \ldots, i_{k} \in\{1, \ldots, n\}$ which is non-decreasing with the possible exception of $i_{0}$, the product $e_{i_{0}} e_{i_{1}} \cdots e_{i_{k}}$ is spanned by terms of the form $e^{a}$ where $|a| \leq k+1$ and

$$
\sum_{i} a_{i} \nu_{i} \geq \nu_{i_{0}}+\cdots+\nu_{i_{k}}
$$

We use double induction, first on $k$ and then on $i_{0}$. The base case $k=0$ is trivial and for given $k$ there is nothing to prove if $i_{0} \leq i_{1}$, so the base case $i_{0}=1$ is trivial. If $i_{0}>i_{1}$, then writing

$$
e_{i_{0}} e_{i_{1}} e_{i_{2}} \cdots e_{i_{k}}=e_{i_{1}} e_{i_{0}} e_{i_{2}} \cdots e_{i_{k}}+\left[e_{i_{0}}, e_{i_{1}}\right] e_{i_{2}} \cdots e_{i_{k}},
$$

we need only prove the claim for both summands on the right hand side. By the induction hypothesis on $k, e_{i_{0}} a_{i_{2}} \cdots e_{i_{k}}$ is a linear combination of $e^{b}$ for $|b| \leq k$ and

$$
\sum_{i} b_{i} \nu_{i} \geq \nu_{i_{0}}+\nu_{i_{2}} \cdots+\nu_{i_{k}}
$$


By the induction hypothesis on $i_{0}, e_{i_{1}}$ times any such $e^{b}$ is a linear combination of terms $e^{a}$ with $|a| \leq k+1$ and $a$ satisfying (3.5).

By (3.1), we can write $\left[e_{i_{0}}, e_{i_{1}}\right]$ as a linear combination of basis vectors $e_{j}$ with $\nu_{j} \geq \nu_{i_{0}}+\nu_{i_{1}}$, so by the induction hypothesis on $k,\left[e_{i_{0}}, e_{i_{1}}\right] e_{i_{2}} \cdots e_{i_{k}}$ is a linear combination of terms of the form $e^{a}$ where $|a| \leq k$ and

$$
\begin{aligned}
\sum_{i} a_{i} \nu_{i} & \geq \nu_{j}+\nu_{i_{2}}+\cdots+\nu_{i_{k}} \\
& \geq \nu_{i_{0}}+\nu_{i_{1}}+\cdots \nu_{i_{k}} .
\end{aligned}
$$

The filtration $\left\{U_{r}\right\}$ of the universal enveloping algebra $U=U(L)$ induces the filtration of the graded Rees algebra $U(L)^{*}$ : we filter each summand $I^{m}$ of $U(L)^{*}$ by the induced filtration given by $U_{\bullet}$ :

$$
F_{r} U(L)^{*}:=\bigoplus_{m=0}^{\infty}\left(I^{m} \cap U_{r}\right)
$$

We get

$$
\operatorname{gr} U(L)^{*}=\bigoplus_{r, m}\left(I^{m} \cap U_{r}\right) /\left(I^{m} \cap U_{r-1}\right)
$$

is a (doubly) graded commutative algebra. We denote

$$
U(L)_{r, m}^{*}=\left(I^{m} \cap U_{r}\right) /\left(I^{m} \cap U_{r-1}\right)
$$

so that $\operatorname{gr} U(L)^{*}=\bigoplus_{r, m} U(L)_{r, m}^{*}$ By the PBW theorem, $U_{1}=L$, so by Lemma 3.3. $U(L)_{1, m}^{*}$ is naturally identified with $L^{m}$.

Proposition 3.4. The graded algebra gr $U(L)^{*}$ is a commutative graded algebra generated by $U(L)_{1, m}^{*}, m=0,1, \ldots, s$, where $L^{s+1}=\{0\}$.

Proof. From Lemma 3.3, we see that $I^{m} \cap U_{r}$ has basis

$$
\left\{e^{a}\left|\sum_{i} a_{i} \nu_{i} \geq m,\right| a \mid \leq r\right\}
$$

so $U(L)_{r, m}^{*}$ has basis

$$
\left\{e^{a}\left|\sum_{i} a_{i} \nu_{i} \geq m,\right| a \mid=r\right\} .
$$

Notice that under the multiplication map

$$
U(L)_{r, m}^{*} \otimes U(L)_{r^{\prime}, m^{\prime}}^{*} \rightarrow U(L)_{r+r^{\prime}, m+m^{\prime}}^{*}
$$

we have $e^{a} \cdot e^{b}=e^{a+b}$. To prove that the classes $U(L)_{1, m}^{*}$ generate, it suffices to note that the additive monoid

$$
\left\{\left(a_{1}, \ldots, a_{n}, r, m\right) \in \mathbb{N}^{n+2}\left|\sum_{i} a_{i} \nu_{i} \geq m,\right| a \mid=r\right\}
$$


is generated by the set

$$
\left\{(\underbrace{0, \ldots, 0}_{i-1}, 1, \underbrace{0, \ldots, 0}_{n-i}, 1, m) \mid 1 \leq i \leq n, 0 \leq m \leq \nu_{i}\right\}
$$

whose elements correspond to $e_{i} \in L^{m}=U(L)_{1, m}^{*} \subset L$.

Now we recall a useful general result. Let $R$ be an associative ring with increasing exhausting filtration

$$
0=F_{-1} \subset F_{0} \subset F_{1} \subset \cdots=R
$$

and consider the associated graded ring

$$
\operatorname{gr} R=\operatorname{gr}_{F_{\bullet}} R=\bigoplus_{n \geq 0} F_{n} / F_{n-1}
$$

If $x \in F_{n} \backslash F_{n-1}$ we say that $x$ has degree $n$ and denote by $\bar{x}$ its image in $F_{n} / F_{n-1}$.

Lemma 3.5. Assume that gr $R$ is graded left Noetherian. Then the ring $R$ is left Noetherian.

Proof. Let $I \subset R$ be a left ideal. Then

$$
\operatorname{gr} I:=\bigoplus_{n \geq 0}\left(I \cap F_{n}\right) /\left(I \cap F_{n-1}\right)
$$

is a graded left ideal in gr $R$. Let $\bar{x}_{1}, \ldots, \bar{x}_{k}$ be a set of homogeneous generators of the ideal gr $I$; say $\operatorname{deg}\left(\bar{x}_{i}\right)=n_{i}$. Choose lifts $x_{1}, \ldots, x_{k} \in I$ of the $\bar{x}_{i}$ 's and let $\left(x_{1}, \ldots, x_{k}\right) \subset I$ be the corresponding left ideal. We claim that $\left(x_{1}, \ldots, x_{k}\right)=I$. Indeed, let $x \in I$ be of degree $d$. If $d=0$, then clearly $x \in\left(x_{1}, \ldots, x_{k}\right)$. Otherwise, there exist $r_{i} \in R$ of degree $d-n_{i}$ such that

$$
\bar{x}=\sum \bar{r}_{i} \bar{x}_{i}
$$

It follows that $x-\sum r_{i} x_{i} \in I \cap F_{d-1}$, hence by induction on $d, x-\sum r_{i} x_{i} \in$ $\left(x_{1}, \ldots, x_{k}\right)$.

It follows from Proposition 3.4 and Lemma 3.5 that the Rees algebra $U(L)^{*}$ is left Noetherian if the Lie algebra is nilpotent. This proves the "if" direction of Theorem 3.1 .

3.2. Proof of the "only if" direction. For a Lie algebra $L$ we consider the lower central series $L_{1}=L, L_{n}=\left[L, L_{n-1}\right]$. Thus

$$
L=L_{1} \supset L_{2} \supset L_{3} \supset \cdots
$$

is a nonincreasing sequence of ideals in $L$. We put

$$
L_{\infty}:=\bigcap_{n} L_{n}, \quad L_{\text {nil }}=L / L_{\infty}
$$

The Lie algebra $L_{\text {nil }}$ is nilpotent, and $L$ is nilpotent if and only if $L_{\infty}=0$.

We have the short exact sequence of Lie algebras

$$
0 \rightarrow L_{\infty} \rightarrow L \rightarrow L_{\text {nil }} \rightarrow 0
$$


This induces the surjection $\theta: U(L) \rightarrow U\left(L_{\text {nil }}\right)$ and $\operatorname{ker} \theta$ is the ideal $U(L) L_{\infty} U(L)$. As before, let $I \subset U(L)$ be the augmentation ideal.

Lemma 3.6. We have the equality of ideals in $U(L)$ :

$$
\bigcap_{n} I^{n}=\operatorname{ker} \theta
$$

Proof. We have by construction $L_{n} \subset I^{n}$ for all $n$, hence $L_{\infty} \subset \bigcap_{n} I^{n}$ and so

$$
\operatorname{ker} \theta=U(L) L_{\infty} U(L) \subset \bigcap_{n} I^{n}
$$

To prove the opposite inclusion let $\bar{I} \subset U\left(L_{\mathrm{nil}}\right)$ be the augmentation ideal, so that we have the surjection $\theta: I \rightarrow \bar{I}$. It suffices to prove that $\bigcap_{n} \bar{I}^{n}=0$. This follows from Lemma 3.3 .

Assume that the Lie algebra $L$ is not nilpotent, i.e. $L_{\infty} \neq 0$. Then Lemma 3.6 implies that $L_{\infty} \subset \bigcap_{n} I^{n}$. Fix $0 \neq x \in L_{\infty}$ and consider the graded left ideal

$$
J=\bigoplus_{n} U(L) x_{n} \subset U(L)^{*}
$$

where $x_{n}$ denotes the copy of $x$ in $I^{n}$. We claim that $J$ is not finitely generated. Assume, on the contrary, that

$$
J=\sum_{i} U(L)^{*} f_{i}
$$

for a finite number of homogeneous elements $f_{i} \in U(L) x_{n_{i}}$. Choose $m>n_{i}$ for all $i$. We claim that

$$
x_{m} \notin \sum_{i} U(L)^{*} f_{i}
$$

Indeed, it suffices to notice that $x \notin I x$ : if $x=f x$, then $f=1$, (since $U(L)$ is a domain) and hence $f \notin I$. This completes the proof of Theorem 3.1 .

\section{MAIN THEOREM}

Let $L$ be a finite dimensional Lie algebra, $U(L)$ its universal enveloping algebra, $I \subset U(L)$ the augmentation ideal. As in Subsection 3.2, consider the ideal

$$
L_{\infty}=\bigcap_{n} L_{n} \subset L
$$

and the quotient nilpotent Lie algebra $L_{\text {nil }}=L / L_{\infty}$.

Each cohomology space $H^{i}\left(L_{\infty}, k\right)$ is naturally a $L_{\text {nil-module, so we have }}$ the Hochschild-Serre spectral sequence [HS]:

$$
E_{2}^{p q}=H^{p}\left(L_{\mathrm{nil}}, H^{q}\left(L_{\infty}, k\right)\right) \Rightarrow H^{p+q}(L, k) .
$$


Theorem 4.1. Let $L$ be a finite dimensional Lie algebra over a field $k$. The following conditions are equivalent:

(1) The natural functor

$$
\Phi_{L}: D^{b}\left((U(L)-\bmod )_{I}\right) \rightarrow D_{I}^{b}(U(L)-m o d)
$$

is an equivalence.

(2) The natural map $H^{\bullet}\left(L_{\mathrm{nil}}, k\right) \rightarrow H^{\bullet}(L, k)$ is an isomorphism.

(3) The positive degree cohomology $H^{>0}\left(L_{\infty}, k\right)$ considered as an $L_{\text {nil- }}$ module has no subquotients isomorphic to the trivial module $k$.

Proof. We first notice that the 3 conditions in the theorem hold in case $L$ is nilpotent. Indeed, then $L_{\infty}=0$, so (2) and (3) hold trivially. Also (1) holds by Corollary 3.2

Let now $L$ be general. As in section 3.2 we consider the short exact sequence of Lie algebras

$$
0 \rightarrow L_{\infty} \rightarrow L \rightarrow L_{\text {nil }} \rightarrow 0
$$

and the induced surjection $\theta: U(L) \rightarrow U\left(L_{\text {nil }}\right)$ with the kernel $\operatorname{ker} \theta=$ $\bigcap_{n} I^{n}$ (Lemma 3.6). Also, as in the proof of Lemma 3.6. denote by $\bar{I}$ the augmentation ideal in $U\left(L_{\text {nil }}\right)$. Lemma 3.6 implies that any $U(L)$-module $M$ such that $M=M_{I}$ is actually a $U\left(L_{\text {nil }}\right)$-module (and $M=M_{\bar{I}}$ ). Hence the functor of restriction of scalars

$$
\theta_{*}: U\left(L_{\text {nil }}\right)-\bmod \rightarrow U(L)-\bmod
$$

induces the equivalence of categories

$$
\left(U\left(L_{\mathrm{nil}}\right)-\bmod \right)_{\bar{I}} \stackrel{\sim}{\rightarrow}(U(L) \text {-mod })_{I}
$$

and therefore the equivalence of categories

$$
D^{b}\left(\left(U\left(L_{\text {nil }}\right)-\bmod \right)_{\bar{I}}\right) \stackrel{\sim}{\rightarrow} D^{b}\left((U(L)-\bmod )_{I}\right)
$$

We have the commutative diagram of functors

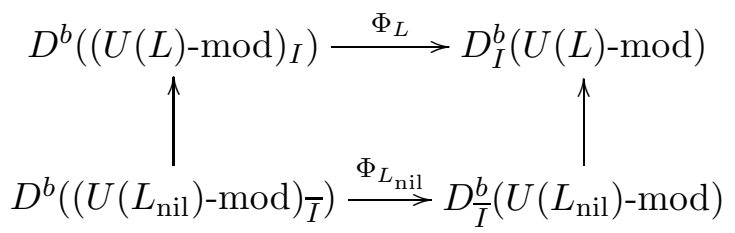

As explained above the left vertical arrow is an equivalence. Also $\Phi_{L_{\mathrm{nil}}}$ is an equivalence (Corollary [3.2). Hence $\Phi_{L}$ is an equivalence if and only if the functor

$$
\theta_{*}: D_{\bar{I}}^{b}\left(U\left(L_{\mathrm{nil}}\right) \text {-mod }\right) \rightarrow D_{I}^{b}(U(L) \text {-mod })
$$

is an equivalence. Every finitely generated $I$-torsion $U(L)$-module (resp. $\bar{I}$-torsion $U\left(L_{\text {nil }}\right)$-module) is a finite dimensional $k$-vector space on which $U(L)$ (resp. $U\left(L_{\text {nil }}\right)$ ) acts nilpotently, so by Engel's theorem, it admits a stable flag with trivial 1-dimensional quotients. As triangulated categories, 
therefore, both sides of (4.4) are generated by the trivial module $k$, and so the functor in (4.4) is an equivalence if and only if the natural map

$$
\theta_{*}: \operatorname{Ext}_{U\left(L_{\text {nil }}\right)}^{\bullet}(k, k) \rightarrow \operatorname{Ext}_{U(L)}^{\bullet}(k, k)
$$

is an isomorphism. This proves the equivalence of conditions (1) and (2) in the theorem. It remains to prove the equivalence of (2) and (3).

First we prove a lemma. Let $\mathcal{L}$ be a nilpotent Lie algebra. By a theorem of Lie every simple $\mathcal{L}$-module $M$ is one dimensional, hence it corresponds to an additive character

$$
\chi_{M}: \mathcal{L} /[\mathcal{L}, \mathcal{L}] \rightarrow k
$$

Lemma 4.2. Let $M$ be a simple nontrivial $\mathcal{L}$-module. Then

$$
H^{\bullet}(\mathcal{L}, M)=0
$$

Proof. The character $\chi_{M}$ gives a short exact sequence of nilpotent Lie algebras

$$
0 \rightarrow \mathcal{L}^{\prime} \rightarrow \mathcal{L} \stackrel{\chi_{M}}{\rightarrow} \overline{\mathcal{L}} \rightarrow 0
$$

with $\operatorname{dim} \overline{\mathcal{L}}=1$, where $\mathcal{L}^{\prime}$ acts trivially on $M$.

Recall [CE, (23.1)] the standard complex $C^{\bullet}\left(\mathcal{L}^{\prime}, M\right)$, which computes the cohomology $H^{\bullet}\left(\mathcal{L}^{\prime}, M\right)$. With our normalization, it is

$$
0 \rightarrow M=C^{0}\left(\mathcal{L}^{\prime}, M\right) \stackrel{\partial_{0}}{\rightarrow} C^{1}\left(\mathcal{L}^{\prime}, M\right) \stackrel{\partial_{1}}{\rightarrow} \cdots,
$$

where $C^{n}\left(\mathcal{L}^{\prime}, M\right):=\operatorname{Hom}_{k}\left(\bigwedge^{n} \mathcal{L}^{\prime}, M\right)$ and

$$
\begin{aligned}
\partial_{n}(f)\left(x_{0} \wedge\right. & \left.\cdots \wedge x_{n}\right)=\sum_{i=0}^{n}(-1)^{i} x_{i} f\left(x_{0} \wedge \cdots \wedge \hat{x}_{i} \wedge \cdots \wedge x_{n}\right) \\
& +\sum_{p<q}(-1)^{p+q} f\left(\left[x_{p}, x_{q}\right] \wedge x_{0} \wedge \cdots \wedge \hat{x}_{p} \wedge \cdots \wedge \hat{x}_{q} \wedge \cdots \wedge x_{n}\right)
\end{aligned}
$$

The $\mathcal{L}$-action on $\mathcal{L}^{\prime}$ and $M$ extends to an action on the complex $C^{\bullet}\left(\mathcal{L}^{\prime}, M\right)$ by the formula

$$
\begin{aligned}
(x f)\left(x_{1} \wedge \cdots \wedge x_{n}\right) & =x f\left(x_{1} \wedge \cdots \wedge x_{n}\right) \\
& -\sum_{i=1}^{n} f\left(x_{1} \wedge \cdots \wedge x_{i-1} \wedge\left[x, x_{i}\right] \wedge x_{i+1} \wedge \cdots \wedge x_{n}\right)
\end{aligned}
$$

This induces the $\mathcal{L}$-action on the cohomology $H^{\bullet}\left(\mathcal{L}^{\prime}, M\right)$ which is trivial on $\mathcal{L}^{\prime}$ and hence gives the required $\bar{L}$-action on $H^{\bullet}\left(\mathcal{L}^{\prime}, M\right)$.

We now take a closer look at the $\mathcal{L}$-action (4.8) on $C^{n}\left(\mathcal{L}^{\prime}, M\right)$. Since the $\mathcal{L}$-action on the Lie algebra $\mathcal{L}^{\prime}$ is nilpotent there exists a basis of $\mathcal{L}^{\prime}$ such that for every $x \in \mathcal{L}$ the matrix of the operator $[x,-]$ in this basis is strictly lower triangular. Then it follows from the formula (4.8) that there exists a basis for $C^{n}\left(\mathcal{L}^{\prime}, M\right)$ such that the action of every $x \in \mathcal{L}$ is given by a lower triangular matrix with all diagonal entries being $\chi_{M}(x)$. 
Therefore any $\bar{x} \in \overline{\mathcal{L}}$ acts on the cohomology $H^{\bullet}\left(\mathcal{L}^{\prime}, M\right)$ by an operator whose characteristic polynomial is a power of $\left(t-\chi_{M}(\bar{x})\right)$. Since we assume that $\chi_{M}(\bar{x}) \neq 0$ for $\bar{x} \neq 0$, it easily follows that

$$
\operatorname{Ext}_{U(\overline{\mathcal{L}})}^{\bullet}\left(k, H^{\bullet}\left(\mathcal{L}^{\prime}, M\right)\right)=H^{\bullet}\left(\overline{\mathcal{L}}, H^{\bullet}\left(\mathcal{L}^{\prime}, M\right)\right)=0
$$

Now the Hochschild-Serre spectral sequence

$$
E_{2}^{p q}=H^{p}\left(\overline{\mathcal{L}}, H^{q}\left(\mathcal{L}^{\prime}, M\right)\right) \Rightarrow H^{p+q}(\mathcal{L}, M)
$$

implies that $H^{\bullet}(\mathcal{L}, M)=0$, which proves the lemma.

We now return to the proof of the equivalence of conditions (2) and (3) in the theorem. The Hochschild-Serre spectral sequence (4.1) has $E_{2}$ page

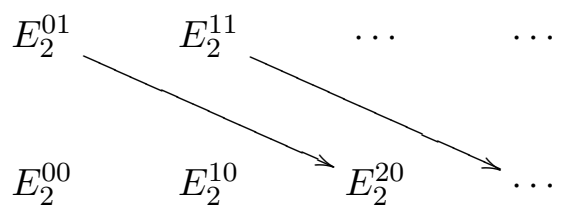

We have $H^{0}\left(L_{\infty}, k\right)=k$ - the trivial $L_{\text {nil-module and the graded space }}$ $\operatorname{Ext}_{U\left(L_{\text {nil }}\right)}(k, k)$ identifies naturally with the bottom row of this spectral sequence. The map $\theta^{*}: \operatorname{Ext}_{U\left(L_{\text {nil }}\right)}^{\bullet}(k, k) \rightarrow \operatorname{Ext}_{U(L)}^{\bullet}(k, k)$ then coincides with the projection

$$
\operatorname{Ext}_{U\left(L_{\mathrm{nil}}\right)}^{\bullet}(k, k)=H^{\bullet}\left(L_{\mathrm{nil}}, H^{0}\left(L_{\infty}, k\right)\right) \rightarrow H^{\bullet}(L, k)
$$

Notice that by a theorem of Lie every finite dimensional $L_{\text {nil-module has }}$ a filtration with 1-dimensional subquotients.

Assume that the condition (3) holds, i.e. the $L_{\text {nil }}$-module $H^{>0}\left(L_{\infty}, k\right)$ has no subquotients isomorphic to the trivial module $k$. Then all the 1dimensional subquotients of the $L_{\text {nil }}$-module $H^{>0}\left(L_{\infty}, k\right)$ are nontrivial. In this case it follows from Lemma 4.2 that only the bottom row of the spectral sequence (4.9) is nonzero. Therefore the natural map $H^{\bullet}\left(L_{\text {nil }}, k\right) \rightarrow$ $H^{\bullet}(L, k)$ is an isomorphism, i.e. the condition (2) of the theorem holds.

Assume, conversely, that condition (2) holds. Let $d$ be the maximal integer such that $H^{d}\left(L_{\text {nil }}, k\right) \neq 0$. Again using Lie's theorem and Lemma 4.2 it follows that $H^{>d}\left(L_{\text {nil }}, N\right)=0$ for any finite dimensional $L_{\text {nil-module }} N$. If $k$ is a subquotient of $N$, then by Engel's theorem, it is also a quotient of $N$. It follows, therefore, that if $N$ has the trivial module as a subquotient,

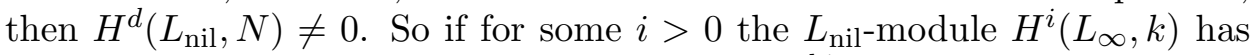
the trivial submodule $k$ as a subquotient then $E_{2}^{d, i}=H^{d}\left(L_{\text {nil }}, H^{i}\left(L_{\infty}, k\right)\right)$ is nonzero, which means that it survives in $H^{i+d}(L, k)$. This is a contradiction and finishes the proof of the theorem.

4.1. Some examples. (A) Consider the 2-dimensional Lie algebra with basis $x, y$ and the relation $[x, y]=y$. This Lie algebra is solvable but not 
nilpotent, $L_{\infty}=k y$. The standard complex, which computes the cohomology $H^{\bullet}\left(L_{\infty}, k\right)$ has terms in degrees 0 and 1 and zero differential

$$
k \stackrel{0}{\rightarrow} \operatorname{Hom}_{k}(k y, k)
$$

The element $x \in L_{\text {nil }}$ acts on the space $\operatorname{Hom}_{k}(k y, k)=H^{1}\left(L_{\infty}, k\right)$ as minus the identity (see formula (4.8)), hence the condition (3) of Theorem 4.1 is satisfied.

(B) This is a generalization of example (A) above: assume that the $L_{\text {nil- }}$ module $\bigwedge^{i} L_{\infty}$ has no subquotients isomorphic to the trivial module $k$ if $i>0$. Then the condition (3) of Theorem 4.1 holds. For example this is the case when $L$ is the Lie algebra of upper-triangular matrices. Then $L_{\infty}$ is the ideal of strictly triangular matrices and $L_{\text {nil }}$ is the abelian quotient.

(C) However, there exists solvable algebras $L$ for which condition (3) does not hold. See Proposition 4.5 below.

(D) It may happen that condition (3) holds even though $\bigwedge^{>0} L_{\infty}$ admits

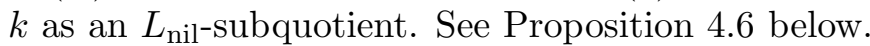

For the next two examples, we assume that $k$ has characteristic zero.

(E) Assume that $L_{\infty} \neq 0$ is semi-simple. If $k=\mathbb{R}$ and $L_{\infty}$ is compact, the cohomology $H^{\bullet}\left(L_{\infty}, k\right)$ is isomorphic to the cohomology of any compact Lie group with the Lie algebra $L_{\infty}$ [CE, Theorem 15.2], so $H^{>0}\left(L_{\infty}, k\right) \neq$ 0 . Since every compact semisimple Lie algebra has a compact real form, $H^{>0}\left(L_{\infty}, k\right) \neq 0$ when $k=\mathbb{C}$ and $L_{\infty}$ is semisimple, the same statement follows for every Lie algebra over any field $k$ of characteristic zero. However for any $x \in L_{\text {nil }}$ the operator $[x,-]$ on of $L_{\infty}$ is a derivation, so is inner. Therefore the action of $L_{\text {nil }}$ of the cohomology is trivial and so the condition (3) of Theorem 4.1 fails.

(F) This is a generalization of example (E) above. We formulate it as a proposition.

Proposition 4.3. Assume that the equivalent conditions of Theorem 4.1 are satisfied and the characteristic of $k$ is zero. Then the algebra $L_{\infty}$ is solvable.

Proof. By the general theory the Lie algebra $L_{\infty}$ has a maximal solvable ideal (the radical) $L_{\infty}^{\mathrm{rad}}$ such that

$$
L_{\infty}^{\beta}:=L_{\infty} / L_{\infty}^{\mathrm{rad}}
$$

is semi-simple. Put $\mathfrak{g}:=L_{\infty}^{\beta}$. We need to prove that $\mathfrak{g}=0$.

Lemma 4.4. In the above notation the natural map

$$
H^{\bullet}(\mathfrak{g}, k)=\operatorname{Ext}_{U(\mathfrak{g})}^{\bullet}(k, k) \rightarrow \operatorname{Ext}_{U\left(L_{\infty}\right)}^{\bullet}(k, k)=H^{\bullet}\left(L_{\infty}, k\right)
$$

is injective.

Proof. By the Levi theorem we know that the surjection of Lie algebras $p: L_{\infty} \rightarrow \mathfrak{g}$ has a splitting $s: \mathfrak{g} \rightarrow L_{\infty}$. The corresponding surjection of universal enveloping algebras $p: U\left(L_{\infty}\right) \rightarrow U(\mathfrak{g})$ induces the pair of adjoint 
functors $\left(L p *, p_{*}\right)$ - the extension and restriction of scalars between the derived categories $D^{b}\left(U\left(L_{\infty}\right)\right.$-mod) and $D^{b}(U(\mathfrak{g})$-mod $)$, where $A$-mod is the category of (all) left modules over an associative ring $A$. It suffices to prove that the adjunction morphism of functors

$$
\operatorname{Id}_{D^{b}(U(\mathfrak{g})-\bmod )} \rightarrow L p^{*} \cdot p_{*}
$$

has a left inverse, i.e. $\operatorname{Id}_{D^{b}(U(\mathfrak{g})-\bmod )}$ is a direct summand of the functor $L p^{*} \cdot p_{*}$.

Let $S^{\bullet}$ be an object in $D^{b}\left(U(\mathfrak{g})\right.$-mod). We may assume that $S^{\bullet}$ consists of projective $U(\mathfrak{g})$-modules. Let us construct a special (functorial) projective resolution of $p_{*} S^{\bullet}$. The morphism $s: \mathfrak{g} \rightarrow L_{\infty}$ gives a homomorphism $s: U(\mathfrak{g}) \rightarrow U\left(L_{\infty}\right)$ such that $p \cdot s=\mathrm{id}$. So we may consider $U\left(L_{\infty}\right)$ as a (free) right $U(\mathfrak{g})$-module via the homomorphism $s$. Consider the obvious short exact sequence of complexes of $U\left(L_{\infty}\right)$-modules

$$
0 \rightarrow K^{\bullet} \rightarrow U\left(L_{\infty}\right) \otimes_{U(\mathfrak{g})} S^{\bullet} \rightarrow p_{*} S^{\bullet} \rightarrow 0
$$

Now we repeat this procedure with $K^{\bullet}$ instead of $S^{\bullet}$ (by first considering $K^{\bullet}$ as a complex of $U(\mathfrak{g})$-modules via the map $s$ ) and so on. Eventually we obtain the complex of (complexes of projective) $U\left(L_{\infty}\right)$-modules

$$
P^{\bullet}:=\cdots \stackrel{\partial_{1}}{\rightarrow} U\left(L_{\infty}\right) \otimes_{U(\mathfrak{g})} K^{\bullet} \stackrel{\partial_{0}}{\rightarrow} U\left(L_{\infty}\right) \otimes_{U(\mathfrak{g})} S^{\bullet}
$$

which is a resolution of $p_{*} S^{\bullet}$ and hence

$$
L p^{*} \cdot p_{*} S^{\bullet}=U(\mathfrak{g}) \otimes_{U\left(L_{\infty}\right)} P^{\bullet}
$$

Note that by construction of $P^{\bullet}$, the map $U(\mathfrak{g}) \otimes_{U\left(L_{\infty}\right)} \partial_{0}$ is zero. Hence $U(\mathfrak{g}) \otimes_{U\left(L_{\infty}\right)}\left(U\left(L_{\infty}\right) \otimes_{U(\mathfrak{g})} S^{\bullet}\right)=S^{\bullet}$ is a direct summand of $L p^{*} \cdot p_{*} S^{\bullet}$, which proves the lemma.

Now the assertion of the proposition follows from the example (E) above. Indeed, if $\mathfrak{g} \neq 0$, then $H^{>0}(\mathfrak{g}, k) \neq 0$. As explained in example (E) the $L_{\text {nil }}$ action on $H^{>0}(\mathfrak{g}, k) \neq 0$ is trivial, so by the above lemma the space $H^{>0}\left(L_{\infty}, k\right)$ contains a nonzero $L_{\text {nil-submodule, which is trivial. This con- }}$ tradicts condition (3) of Theorem 4.1 .

Proposition 4.5. There exist solvable Lie algebras $L$ not satisfying the equivalent conditions of Theorem 4.1.

Proof. For a nilpotent Lie algebra $N$ acting on the trivial module $k$, the top differential, $C^{\operatorname{dim} N-1}(N, k) \rightarrow C^{\operatorname{dim} N}(N, k)$, of the Chevalley-Eilenberg complex is always zero. Therefore, if $L_{\infty}$ is nilpotent and $L_{\text {nil }}$ acts trivially on $\bigwedge^{\operatorname{dim} L_{\infty}} L_{\infty}$, then condition (3) of Theorem 4.1 is violated.

Let $L$ be the subalgebra of upper-triangular $3 \times 3$ matrices such that the upper left and lower right entries are the same. Thus $L_{\infty}$ consists of strictly upper triangular matrices, and its center $Z$ consists of matrices which are zero except possibly in the upper right entry. The commutator map $L_{\infty} / Z \times L_{\infty} / Z \rightarrow Z$ respects the action of $L_{\text {nil }}$, which is trivial on $Z$. This 
implies that $\bigwedge^{2}\left(L_{\infty} / Z\right) \cong k$ as $L_{\text {nil }}$-module, and therefore $\bigwedge^{3} L_{\infty} \cong k$ as $L_{\text {nil-module. }}$

Proposition 4.6. The conditions of Theorem 4.1 are strictly weaker than

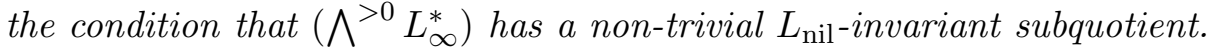

Proof. As $H^{>0}\left(L_{\infty}, k\right)$ is a subquotient of $\bigwedge^{>0} L_{\infty}^{*}$, if the former has a nontrivial $L_{\text {nil }}$-invariant subquotient, the latter does as well.

We show that converse does not hold by exhibiting a case in which $L_{\text {nil }}$ acts semisimply on $\bigwedge^{\bullet} L_{\infty}^{*}$ and therefore on every $L_{\text {nil-stable subquotient }}$ and for which

$$
\operatorname{dim}\left(\bigwedge^{\bullet} L_{\infty}^{*}\right)^{L_{\text {nil }}}>1=\operatorname{dim} H^{\bullet}\left(L_{\infty}, k\right)^{L_{\text {nil }}} .
$$

The free Lie algebra on two generators $x$ and $y$ admits a unique bigrading for which $x$ and $y$ have bidegree $(1,0)$ and $(0,1)$ respectively. Let $M$ be the quotient of this algebra by the graded ideal generated by all elements of total degree $\geq 4$ and also $[[x, y], y]$. Then $M$ has basis: $x, y, z, w$ of bidegree $(1,0),(0,1),(1,1)$, and $(2,1)$ respectively, satisfying the following relations:

$$
[x, y]=z,[x, z]=w,[x, w]=[y, z]=[y, w]=[z, w]=0
$$

(see [Bo, II, $\S 2$, no. 11, Théorème 1] and the computation of the Hall set for 2 generators given at the end of no. 10.)

We define $t$ to be the derivation which acts on the bidegree $(a, b)$ part of $M$ by $2 a-3 b$. Let $L:=M \oplus k t$ denote the semi-direct sum, so

$$
[t, x]=2 x,[t, y]=-3 y,[t, z]=-z,[t, w]=w .
$$

We confirm that $[L, L]=[L, M]=M$, so $L_{\infty}=M$, and $L_{\text {nil }}$ is the 1dimensional algebra spanned by the class of $t$.

Next, we consider the Chevalley-Eilenberg complex of $M$. The underlying graded space is $\bigwedge^{\bullet} M^{*}$, which is spanned by wedge products of the dual basis $x^{*}, y^{*}, z^{*}, w^{*}$ of $M$. The differential is given by

$$
\delta\left(x^{*}\right)=0, \delta\left(y^{*}\right)=0, \delta\left(z^{*}\right)=y^{*} \wedge x^{*}, \delta\left(w^{*}\right)=z^{*} \wedge x^{*} .
$$

The bigrading on $M$ induces a bigrading on $\wedge^{\bullet} M^{*}$, and $\delta$ preserves bidegree. The degree $(3,2)$-part of $\wedge^{\bullet} M^{*}$ is spanned by $z^{*} \wedge w^{*}$ and $x^{*} \wedge y^{*} \wedge w^{*}$. As

$$
\delta\left(z^{*} \wedge w^{*}\right)=-x^{*} \wedge y^{*} \wedge z^{*},
$$

the degree $(3,2)$-part of $H^{*}\left(L_{\infty}, k\right)$ is zero. On the other hand, the $t$ invariant part of $H^{*}\left(L_{\infty}, k\right)$ is the sum of the $(3 n, 2 n)$-part over all integers $n$.

Now $H^{*}\left(L_{\infty}, k\right)$ is a subquotient of $\bigwedge^{\bullet} M^{*}$, and the latter has non-trivial degree $(3 n, 2 n)$-part only for $n=0,1$. Thus, $\operatorname{dim}\left(\bigwedge^{\bullet} L_{\infty}\right)^{L_{\text {nil }}}=3$ but

$$
\operatorname{dim} H^{\bullet}\left(L_{\infty}, k\right)^{L_{\text {nil }}}=1 .
$$




\section{An APPLICATION}

Let $k$ be an algebraically closed field of characteristic zero. Let $V$ be a linear unipotent algebraic group over $k, L=$ Lie $V$ the corresponding nilpotent Lie algebra.

Denote by $V$-Mod the abelian category of rational representations of $V$. Recall that an object of $V$-mod is by definition a $V$-module $M$ which is a union of finite dimensional submodules $M_{i}$, such that the $V$-action on $M_{i}$ comes from a homomorphism of $k$-algebraic groups $V \rightarrow G L\left(M_{i}\right)$. In particular every element of $V$ acts on $M_{i}$ via a unipotent operator.

Notice that we have a natural equivalence of abelian categories

$$
\log : V \text {-Mod } \rightarrow(U(L)-\operatorname{Mod})_{I}
$$

where $(U(L) \text {-Mod })_{I}$ is the abelian category of (all) $U(L)$-modules which are $I$-torsion.

This induces the equivalence of derived categories

$$
\log : D^{b}(V \text {-Mod }) \rightarrow D^{b}\left((U(L)-\operatorname{Mod})_{I}\right)
$$

Recall that for $M \in V$-mod its cohomology is by definition

$$
H_{V}^{\bullet}(M):=\operatorname{Ext}_{V \text {-Mod }}^{\bullet}(k, M)
$$

where $k$ is the trivial rational $V$-module.

Corollary 5.1. For any $M \in V$-Mod we have the isomorphism

$$
H_{V}^{\bullet}(M) \simeq H^{\bullet}(L, \log (M))
$$

In particular, the cohomology $H_{V}^{\bullet}(M)$ can be computed using the standard complex for the Lie algebra $L$.

Proof. The equivalence (5.1) implies the isomorphism

$$
\operatorname{Ext}_{V \text {-Mod }}^{\bullet}(k, M)=\operatorname{Ext}_{(U(L) \text {-Mod })_{I}}^{\bullet}(k, \log (M))
$$

The module $M$ is a direct limit (union) of its finite dimensional submodules. The cohomology on both sides of (5.2) commutes with direct limits, hence we may assume that $\operatorname{dim}_{k} M<\infty$ and so the $\log (M) \in U(L)$-mod. Using the standard methods one can show that

$$
\operatorname{Ext}_{(U(L)-\operatorname{Mod})_{I}}^{\bullet}(k, \log (M))=\operatorname{Ext}_{(U(L)-\bmod )_{I}}^{\bullet}(k, \log (M))
$$

Finally, Corollary 3.2 implies the isomorphism

$$
\operatorname{Ext}_{(U(L)-\bmod )_{I}}^{\bullet}(k, \log (M))=\operatorname{Ext}_{U(L)-\bmod }^{\bullet}(k, \log (M))
$$

which proves the corollary.

Let $X$ be a $k$-scheme with an action of the group $V$. For a $V$-equivariant quasi-coherent sheaf $F$, its cohomology can be computed as

$$
H_{V}^{\bullet}(X, F)=\operatorname{Ext}_{V \text {-Mod }}^{\bullet}(k, \mathbb{R} \Gamma(X, F)),
$$

and sometimes one wants to know that the Ext-space $\operatorname{Ext}_{V \text {-Mod }}^{\bullet}(k,-)$ can be computed using the standard complex for the Lie algebra $L$ (by Corollary 
5.1). This fact was used, for example, in the key computation on p. 8 of [Te].

\section{REFERENCES}

[Bo] N. Bourbaki, Éléments de mathématique. Fasc. XXXVII. Groupes et algèbres de Lie. Chapitre II: Algèbres de Lie libres. Chapitre III: Groupes de Lie. Actualités Scientifiques et Industrielles, No. 1349. Hermann, Paris, 1972.

[CE] C. Chevalley and S. Eilenberg, Theory of Lie groups and Lie algebras. Trans. Amer. Math. Soc. 63 (1948), 85-124.

[HS] G. Hochschild and J-P. Serre, Cohomology of Lie algebras, Annals of Math. 57 (1953), 591-603.

[Te] C. Teleman, The quantization conjecture revisited, Annals of Math. 152, no. 1, (2000), 1-43.

Department of Mathematics, Indiana University, Bloomington, IN 47405 , U.S.A.

Department of Mathematics, Indiana University, Bloomington, IN 47405, U.S.A.; HSE UNIVERSITY, Russian FEDERATION 